\title{
О ДВУСТОРОННИХ АППРОКСИМАЦИЯХ МНОЖКСТВ ДОСТИЖИМОСТИ УПРАВЛЯЕМЫХ СИСТЕМ С ГЕОМЕТРИЧЕСКИМИ ОГРАНИЧЕНИЯМИ НА УПРАВЛЕНИЯ ${ }^{1}$
}

\author{
В. Н. Ушаков, М. В. Першаков
}

\begin{abstract}
Рассматривается нелинейная управляемая система в евклидовом пространстве и на конечном промежутке времени, управления которой стеснены геометрическими ограничениями. Изучается вопрос о конструировании нижних и верхних (по включению) аппроксимаций множеств достижимости управляемой системы. При определенных условиях на систему получены оценки рассогласования (в хаусдорфовой метрике) между нижними и верхними аппроксимациями множеств достижимости.

Ключевые слова: управляемая система, управление, дифференциальное включение, геометрические ограничения, множество достижимости, аппроксимация.
\end{abstract}

V. N. Ushakov, M. V. Pershakov. On two-sided approximations of reachable sets of control systems with geometric constraints on the controls.

We consider a nonlinear control system in Euclidean space on a finite time interval with controls subject to geometric constraints. The question of constructing lower and upper (by inclusion) approximations of reachable sets of this system is studied. Under certain conditions, estimates are obtained for the discrepancy (in the Hausdorff metric) between the lower and upper approximations of the reachable sets.

Keywords: control system, control, differential inclusion, geometric constraints, reachable set, approximation.

MSC: 37C50, 37J25, 41A29

DOI: $10.21538 / 0134-4889-2020-26-1-239-255$

\section{Введение}

В работе рассматривается нелинейная управляемая система в конечномерном евклидовом пространстве, управления которой стеснены геометрическими ограничениями. На систему наложены достаточно общие условия. Одна из наиболее актуальных тем в теории управления динамическими системами посвящена изучению их множеств достижимости, а также вопросам и задачам, относящимся к конструированию и оценкам этих множеств [1-3]. С этой тематикой сопряжена тематика разнообразных задач о наведении динамических систем на целевые множества и игровых задачах управления [4;5].

В работе изучается вопрос о конструировании и оценках множеств достижимости и трубок траекторий управляемых систем. История этого вопроса насчитывает не одно десятилетие. При этом параллельно развивались как теория, описывающая эволюцию множеств достижимости, так и методы конструирования и оценки этих множеств. Для некоторых достаточно представительных классов систем с различными типами ограничений на управления предложены эффективные методы и алгоритмы конструирования множеств достижимости и трубок траекторий управляемых систем. Параллельно развивалась теория и предлагались схемы для приближенного вычисления множеств достижимости дифференциальных включений (см., например, [6]). K наиболее продвинутым в упомянутых направлениях методам относятся методы, базирующиеся на эллипсоидальном оценивании [7;8]. Отметим, в частности, относящуюся

\footnotetext{
${ }^{1}$ Исследование выполнено при финансовой поддержке РФФИ в рамках научных проектов №18-0100264 и 18-01-00221.
} 
непосредственно к тематике настоящей работы статью [9], в которой были изучены дифференциальные уравнения, описывающие эволюцию эллипсоидов, аппроксимирующих снаружи и изнутри множества достижимости управляемых систем, линейных по фазовой переменной. Изучение множеств достижимости управляемых систем и дифференциальных включений не ограничивается, однако, лишь вопросами, связанными с конструированием и оценкой этих множеств. Очень важным для построения оптимальных управлений в задачах оптимального управления является изучение структуры этих множеств и их границы, а также изучение свойств их граничных точек. Так, экстремальные свойства граничных точек множеств достижимости нелинейных систем с интегральными ограничениями на управления и алгоритмы построения этих множеств, использующие принцип максимума Понтрягина, изучались в [10].

Еще одна весомая группа методов основана на дискретизации времени (см., например, [11]) и использовании полиэдральных представлений [12] и, в том числе, пиксельных представлений аппроксимирующих множеств [13;14]. К работам, упомянутым в последней группе, близки работа [15], в которой получена внешняя оценка множества достижимости нелинейной многошаговой динамической системы, и работа [16], посвященная решению задачи о сближении нелинейных управляемых систем с целевым компактным множеством в фазовом пространстве.

В настоящей статье для нелинейной управляемой системы достаточно общего вида в конечномерном евклидовом пространстве и с геометрическими ограничениями на управления изучаются вопросы, относящиеся к конструированию нижних и верхних (по включению) аппроксимаций множеств достижимости управляемой системы. При получении оценок рассогласования нижних аппроксимаций и (идеального) множества достижимости применена важная оценка Л. С. Понтрягина геометрической разности выпуклого полноразмерного компакта в $\mathbb{R}^{m}$ и замкнутого шара достаточно малого радиуса, полученная им в [5] при изучении линейных дифференциальных игр.

\section{1. Управляемая система на конечном промежутке времени}

На промежутке времени $\left[t_{0}, \theta\right], t_{0}<\theta<\infty$, задана управляемая система

$$
\begin{gathered}
\frac{d x}{d t}=f(t, x, u), \\
u=u(t) \in P ;
\end{gathered}
$$

здесь $t$ - время, $x$ - фазовый вектор системы из $\mathbb{R}^{m}, u$ - вектор управляющих воздействий, $P \in \operatorname{comp}\left(\mathbb{R}^{r}\right)$ - метрическое пространство в евклидовом пространстве $\mathbb{R}^{r}$ с хаусдорфовой метрикой.

Выполнены следующие условия на систему (1.1).

А. Вектор-функция $f(t, x, u)$ определена и непрерывна на $\left[t_{0}, \theta\right] \times \mathbb{R}^{m} \times P$, и для любого компакта $D \subset\left[t_{0}, \theta\right] \times \mathbb{R}^{m}$ найдется такая постоянная $L=L_{D} \in(0, \infty)$, что

$$
\left\|f\left(t, x^{(1)}, u\right)-f\left(t, x^{(2)}, u\right)\right\| \leq L\left\|x^{(1)}-x^{(2)}\right\|, \quad\left(t, x^{(i)}\right) \in D, \quad i=1,2, \quad u \in P ;
$$

здесь $\|f\|$ - норма вектора $f$ в пространстве $\mathbb{R}^{m}$.

В. Существует такая постоянная $\varkappa \in(0, \infty)$, что

$$
\|f(t, x, u)\| \leq \varkappa(1+\|x\|), \quad(t, x, u) \in\left[t_{0}, \theta\right] \times \mathbb{R}^{m} \times P .
$$

C. Для любой точки $(t, x) \in\left[t_{0}, \theta\right] \times \mathbb{R}^{m}$ справедливо $\operatorname{dim} F(t, x)=m$; здесь $F(t, x)=$ $\operatorname{co} \mathcal{F}(t, x), \mathcal{F}(t, x)=\{f(t, x, u): u \in P\}, \operatorname{dim} F-$ топологическая размерность множества $F$, со $F$ - выпуклая оболочка множества $F$ в $\mathbb{R}^{m}$. 
Управляемой системе (1.1) поставим в соответствие дифференциальное включение (д.в.) на $\left[t_{0}, \theta\right]$

$$
\frac{d x}{d t}=F(t, x)
$$

Из условия А следует, что для любого компакта $D \subset\left[t_{0}, \theta\right] \times \mathbb{R}^{m}$ справедливо

$$
K=K_{D}=\max \{\|f(t, x, u)\|:(t, x, u) \in D \times P\}<\infty
$$

и функция

$$
\omega_{D}^{*}(\rho)=\max _{\substack{\left(t_{*}, x_{*}\right) \in D,\left(t^{*}, x^{*}\right) \in D}}\left\{\left\|f\left(t_{*}, x_{*}, u\right)-f\left(t^{*}, x^{*}, u\right)\right\|:\left|t_{*}-t^{*}\right|+\left\|x_{*}-x^{*}\right\| \leq \rho, u \in P\right\}, \quad \rho \in(0, \infty),
$$

удовлетворяет соотношению $\omega_{D}^{*}(\rho) \downarrow 0$ при $\rho \downarrow 0$.

Введем обозначения для различных множеств достижимости (м.д.) системы (1.1) и д.в. (1.2) (пусть $t_{0} \leq t_{*}<t^{*} \leq \theta, x_{*} \in \mathbb{R}^{m}, X_{*} \subset \mathbb{R}^{m}$ ):

$X\left(t^{*}, t_{*}, x_{*}\right)$ - м.д. системы (1.1) в момент $t^{*}$, где $x\left(t_{*}\right)=x_{*}$ - начальная точка;

$X\left(t^{*}, t_{*}, X_{*}\right)$ - м.д. системы (1.1) в момент $t^{*}$, где $X_{*}$ - начальное множество;

$Y\left(t^{*}, t_{*}, x_{*}\right)$ - м.д. Д.в. (1.2) в момент $t^{*}$, где $x\left(t_{*}\right)=x_{*}$ - начальная точка;

$Y\left(t^{*}, t_{*}, X_{*}\right)$ - м.д. д.в. (1.2) в момент $t^{*}$, где $X_{*}$ - начальное множество.

В случае $X_{*} \in \operatorname{comp}\left(\mathbb{R}^{m}\right)$ справедливо $Y\left(t^{*}, t_{*}, X_{*}\right)=\operatorname{cl} X\left(t^{*}, t_{*}, X_{*}\right)$; здесь cl $X$ - замыкание множества $X$ в $\mathbb{R}^{m}$.

Полагаем, что наряду с системой (1.1) задано стартовое множество $X_{0} \in \operatorname{comp}\left(\mathbb{R}^{m}\right)$ для системы (1.1) (для д.в. (1.2)), соответствующее моменту $t_{0}$.

Из условий $\mathbf{A}, \mathbf{B}$ вытекает, что существует такой достаточно большой шар $\Omega=B\left(0 ; R^{0}\right)$, $R^{0} \in(0, \infty)$, где цилиндрическая область $\mathcal{D}=\left[t_{0}, \theta\right] \times \Omega$ удовлетворяет соотношению

$$
Y\left(t, t_{0}, X_{0}\right)_{\varepsilon^{*}} \subset \mathcal{D}, t \in\left[t_{0}, \theta\right]
$$

здесь $B(z ; r)=\left\{b \in \mathbb{R}^{m}:\|b-z\| \leq r\right\} \quad\left(z \in \mathbb{R}^{m}: r \in(0, \infty)\right), \varepsilon^{*} \in(0, \infty), Y_{\varepsilon}$ - замкнутая $\varepsilon$-окрестность множества $Y$ в $\mathbb{R}^{m}$.

Именно эта область $\mathcal{D}$ используется далее в наших рассуждениях; с ней мы связываем постоянные $L=L_{\mathcal{D}}, K=K_{\mathcal{D}}$ и функцию $\omega^{*}(\rho)=\omega_{\mathcal{D}}^{*}(\rho), \rho \in(0, \infty)$.

В настоящей работе рассмотрим вопросы, относящиеся к конструированию нижних и верхних (по включению) аппроксимаций м.д. $Y\left(t, t_{0}, X_{0}\right), t \in\left[t_{0}, \theta\right]$ д.в. (1.2), поскольку их точное вычисление, как правило, не представляется возможным. Также обсудим вопросы, относящиеся к получению оценки хаусдорфова рассогласования (расстояния) между нижними и верхними аппроксимациями множеств $Y\left(t, t_{0}, X_{0}\right), t \in\left[t_{0}, \theta\right], X_{0} \in \operatorname{comp}\left(\mathbb{R}^{m}\right)$.

Предварительно сформулируем утверждение, которым воспользуемся при оценке рассогласования между нижними и верхними аппроксимациями множеств $Y\left(t, t_{0}, X_{0}\right), t \in\left[t_{0}, \theta\right]$.

Для этого введем вспомогательные понятия.

Пусть $F \in \operatorname{comp}\left(\mathbb{R}^{m}\right)$ - выпуклое множество с $\operatorname{dim} F=m$ и $\varepsilon \in(0, \infty)$.

Под вписанным в компакт $F$ замкнутым шаром в $\mathbb{R}^{m}$ понимаем шар максимального радиуса, содержащийся в $F$.

Радиус $r_{F}$ вписанного в $F$ шара определяется равенством $r_{F}=\underset{\substack{w \in F \\ r>0}}{\max }\{r: B(w ; r) \subset F\}$.

Коэффициентом вытянутости множества $F$ назовем число $\gamma_{F}=\frac{d_{F}}{r_{F}}$, где $d_{F}$ - диаметр множества $F, r_{F}$ - радиус замкнутого шара в $\mathbb{R}^{m}$, вписанного в $F$.

Полагая $\varepsilon \in\left(0, r_{F}\right)$, введем множества

$$
\begin{aligned}
& F_{\varepsilon}=F+B(0 ; \varepsilon)=\left\{f \in \mathbb{R}^{m}: \rho(f ; F) \leq \varepsilon\right\},
\end{aligned}
$$

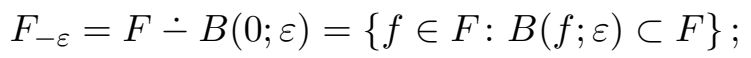


здесь $F+B(0 ; \varepsilon)$ - сумма Минковского множеств $F$ и $B(0 ; \varepsilon), \quad F \dot{-} B(0 ; \varepsilon)-$ разность Минковского (геометрическая разность) множеств $F$ и $B(0 ; \varepsilon)$ (см. [5, с. 314]).

Заметим, что $F_{\varepsilon}$ и $F_{-\varepsilon}-$ выпуклые компакты в $\mathbb{R}^{m}$.

Вопрос о хаусдорфовом отклонении выпуклого компакта в $\mathbb{R}^{m}$ от геометрической разности этого компакта и шара в $\mathbb{R}^{m}$ рассматривался ранее Л. С. Понтрягиным в статье [5, с. 314] при изучении линейных дифференциальных игр. В ней Л. С. Понтрягин получил оценку сверху упомянутой геометрической разности. В данной работе мы применим эту оценку при изучении нелинейной управляемой системы на конечном промежутке времени. Она вплетена в утверждение, которым воспользуемся в последующем при выводе оценок сверху хаусдорфова рассогласования между нижними и верхними аппроксимациями множеств достижимости $Y\left(t, t_{0}, X_{0}\right), t \in\left[t_{0}, \theta\right], X_{0} \in \operatorname{comp}\left(\mathbb{R}^{m}\right)$.

Утверждение. Пусть выпуклые компакты $F u \Phi$ в $\mathbb{R}^{m}$ и число $\varepsilon$ таковы, что $\varepsilon \in(0, \infty)$, $\operatorname{dim} F=m u d(F, \Phi) \leq \varepsilon$. Тогда

$$
h\left(F, F_{-\varepsilon}\right) \leq \gamma_{F} \varepsilon, \quad F_{-\varepsilon} \subset \Phi \subset F_{\varepsilon} ;
$$

здесь $h\left(F_{*}, F^{*}\right)=\max _{f_{*} \in F_{*}} \min _{f^{*} \in F^{*}}\left\|f_{*}-f^{*}\right\|, d\left(F_{*}, F^{*}\right)=\max \left(h\left(F_{*}, F^{*}\right), h\left(F^{*}, F_{*}\right)\right)-$ хаусдорфово расстояние между компактами $F_{*}$ и $F^{*}$ в $\mathbb{R}^{m}$.

Обратимся теперь к многозначному отображению $(t, x) \mapsto F(t, x),(t, x) \in \mathcal{D}$.

В силу условия $\mathbf{A}$ это отображение непрерывно (в метрике Хаусдорфа) на компакте $\mathcal{D}$ и, кроме того, согласно условию $\mathbf{C}$, выполняется $r_{F(t, x)}>0,(t, x) \in \mathcal{D}$. Отсюда вытекает, что скалярное отображение $(t, x) \mapsto r_{F(t, x)}>0$ непрерывно (в метрике Хаусдорфа) на $\mathcal{D}$ и, следовательно,

$$
\min _{(t, x) \in \mathcal{D}} r_{F(t, x)}=r_{*}>0 .
$$

Это означает, что для любой точки $(t, x) \in \mathcal{D}$ найдется шар $B\left(z(t, x) ; r_{*}\right)$ в $\mathbb{R}^{m}$ такой, что

$$
B\left(z(t, x) ; r_{*}\right) \subset F(t, x) .
$$

Учитывая также непрерывность (в метрике Хаусдорфа) скалярного отображения $(t, x) \mapsto$ $d_{F(t, x)}$ на $\mathcal{D}$, получаем

$$
\max _{(t, x) \in \mathcal{D}} d_{F(t, x)}=d_{*}<\infty .
$$

В результате выводим, что функция $(t, x) \mapsto \gamma_{F(t, x)}$ ограничена сверху на $\mathcal{D}$, а именно

$$
\max _{(t, x) \in \mathcal{D}} \gamma_{F(t, x)} \leq \gamma_{*}=\frac{d_{*}}{r_{*}}<\infty
$$

Пусть $\left(t_{*}, x_{*}\right) \in \mathcal{D}, t_{0} \leq t_{*}<t^{*} \leq \theta, \delta=t^{*}-t_{*}>0$ и $x(t), x\left(t_{*}\right)=x_{*},-$ решение д.в. (1.2) на промежутке $\left[t_{*}, t^{*}\right]$.

Для решения $x(t)$ при $t \in\left[t_{*}, t^{*}\right]$ справедливо неравенство

$$
\omega^{*}\left(\left|t-t_{*}\right|+\left\|x(t)-x_{*}\right\|\right) \leq \omega^{*}\left(\left|t-t_{*}\right|+\left\|\int_{t_{*}}^{t} f(\tau) d \tau\right\|\right) \leq \omega^{*}((1+K) \delta)=\varphi(\delta) ;
$$

здесь $\frac{d x(t)}{d t} \in F(t, x(t))$ п.в. на $\left[t_{*}, t^{*}\right]$.

Из (1.3) с учетом условия $\mathbf{A}$ получаем для решения $x(t), t \in\left[t_{*}, t^{*}\right]$, д.в. (1.2)

$$
d\left(F(t, x(t)), F\left(t_{*}, x_{*}\right)\right) \leq \varphi(\delta), t \in\left[t_{*}, t^{*}\right] .
$$

В дополнение к условию $\delta=t^{*}-t_{*}>0$ полагаем $\delta>0$ настолько малым, что $\varphi(\delta)<r_{*}$. 
Полагаем при $\left(t_{*}, x_{*}\right) \in \mathcal{D},\left(t_{*}, X_{*}\right) \subset \mathcal{D}$ и указанном $\delta>0$ :

$F\left(t_{*}, x_{*}\right)_{-\varphi(\delta)}=F\left(t_{*}, x_{*}\right)-B(0 ; \varphi(\delta))$;

$F\left(t_{*}, x_{*}\right)_{\varphi(\delta)}=F\left(t_{*}, x_{*}\right)+B(0 ; \varphi(\delta))$

$\widetilde{Y}\left(t^{*}, t_{*}, x_{*}\right)=x_{*}+\delta F\left(t_{*}, x_{*}\right)$;

$\widetilde{Y}\left(t^{*}, t_{*}, X_{*}\right)=\bigcup_{x_{*} \in X_{*}} \tilde{Y}\left(t^{*}, t_{*}, x_{*}\right)$

$\widetilde{Y}^{-}\left(t^{*}, t_{*}, x_{*}\right)=\widetilde{Y}\left(t^{*}, t_{*}, x_{*}\right)-B(0 ; \omega(\delta))=x_{*}+\delta F\left(t_{*}, x_{*}\right)_{-\varphi(\delta)} ;$

$\widetilde{Y}^{-}\left(t^{*}, t_{*}, X_{*}\right)=\bigcup_{x_{*} \in X_{*}} \widetilde{Y}^{-}\left(t^{*}, t_{*}, x_{*}\right)$;

$\widetilde{Y}^{+}\left(t^{*}, t_{*}, x_{*}\right)=\widetilde{Y}\left(t^{*}, t_{*}, x_{*}\right)+B(0 ; \omega(\delta))=x_{*}+\delta F\left(t_{*}, x_{*}\right)_{\varphi(\delta)} ;$

$\widetilde{Y}^{+}\left(t^{*}, t_{*}, X_{*}\right)=\bigcup_{x_{*} \in X_{*}} \tilde{Y}^{+}\left(t^{*}, t_{*}, x_{*}\right)$; здесь $\omega(\delta)=\delta \cdot \varphi(\delta), \delta \in(0, \infty)$.

При условиях, наложенных на $\delta>0$, множества $\widetilde{Y}^{-}\left(t^{*}, t_{*}, x_{*}\right), \widetilde{Y}^{-}\left(t^{*}, t_{*}, X_{*}\right)$ непусты и тем более непусты множества $\widetilde{Y}\left(t^{*}, t_{*}, x_{*}\right), \widetilde{Y}\left(t^{*}, t_{*}, X_{*}\right), \widetilde{Y}^{+}\left(t^{*}, t_{*}, x_{*}\right), \widetilde{Y}^{+}\left(t^{*}, t_{*}, X_{*}\right)$.

Принимая во внимание (1.4) и утверждение, сформулированное выше, и считая, что в этой лемме $F=F\left(t_{*}, x_{*}\right), \Phi=F(t, x(t)), \varepsilon=\varphi(\delta)$, имеем

$$
F\left(t_{*}, x_{*}\right)_{-\varphi(\delta)} \subset F(t, x(t)) \subset F\left(t_{*}, x_{*}\right)_{\varphi(\delta)} .
$$

Из (1.5) следуют включения

$$
\begin{aligned}
& \tilde{Y}^{-}\left(t^{*}, t_{*}, x_{*}\right) \subset Y\left(t^{*}, t_{*}, x_{*}\right) \subset \tilde{Y}^{+}\left(t^{*}, t_{*}, x_{*}\right), \\
& \tilde{Y}^{-}\left(t^{*}, t_{*}, x_{*}\right) \subset \widetilde{Y}\left(t^{*}, t_{*}, x_{*}\right) \subset \widetilde{Y}^{+}\left(t^{*}, t_{*}, x_{*}\right) .
\end{aligned}
$$

Из включений (1.6) следуют включения

$$
\begin{aligned}
& \widetilde{Y}^{-}\left(t^{*}, t_{*}, X_{*}\right) \subset Y\left(t^{*}, t_{*}, X_{*}\right) \subset \widetilde{Y}^{+}\left(t^{*}, t_{*}, X_{*}\right), \\
& \widetilde{Y}^{-}\left(t^{*}, t_{*}, X_{*}\right) \subset \widetilde{Y}\left(t^{*}, t_{*}, X_{*}\right) \subset \widetilde{Y}^{+}\left(t^{*}, t_{*}, X_{*}\right) .
\end{aligned}
$$

\section{2. Нижние и верхние аппроксимации множеств достижимости $Y\left(t_{i}, t_{0}, X_{0}\right), t_{i} \in \Gamma$ д.в. (1.2) и оценка их рассогласования}

В этом разделе введем нижние и верхние аппроксимации множества достижимости д.в. $(1.2)$, отвечающие моментам конечного разбиения $\Gamma$ промежутка $\left[t_{0}, \theta\right]$ и получим оценку сверху рассогласования (в хаусдорфовой метрике) между этими аппроксимациями.

Итак, введем разбиение $\Gamma=\left\{t_{0}, t_{1}, \ldots, t_{i}, \ldots, t_{N}=\theta\right\}$ промежутка $\left[t_{0}, \theta\right]$ и множества в $\mathbb{R}^{m}$, определяемое следующими рекуррентными соотношениями:

$$
\begin{aligned}
& \widetilde{Y}^{-}\left(t_{0}\right)=\widetilde{Y}\left(t_{0}\right)=\widetilde{Y}^{+}\left(t_{0}\right)=Y\left(t_{0}\right)=X_{0} ; \\
& Y\left(t_{i}\right)=Y\left(t_{i}, t_{i-1}, Y\left(t_{i-1}\right)\right) ; \\
& \widetilde{Y}^{-}\left(t_{i}\right)=\widetilde{Y}^{-}\left(t_{i}, t_{i-1}, \widetilde{Y}^{-}\left(t_{i-1}\right)\right) ; \\
& \widetilde{Y}^{+}\left(t_{i}\right)=\widetilde{Y}^{+}\left(t_{i}, t_{i-1}, \widetilde{Y}^{+}\left(t_{i-1}\right)\right) ; \\
& \widetilde{Y}\left(t_{i}\right)=\widetilde{Y}\left(t_{i}, t_{i-1}, \widetilde{Y}\left(t_{i-1}\right)\right) ; i=\overline{1, N-1} .
\end{aligned}
$$

Предполагаем, что диаметр $\Delta=\Delta(\Gamma)=\Delta_{i}=t_{i-1}-t_{i}, i=\overline{1, N-1}$ разбиения $\Gamma$ удовлетворяет неравенству $\varphi(\Delta)<r_{*}$.

При этом предположении будет выполнено условие $\widetilde{Y}^{-}\left(t_{i}\right) \neq \varnothing, i=\overline{1, N-1}$. Тем более будут непусты множества $Y\left(t_{i}\right), \widetilde{Y}\left(t_{i}\right), \widetilde{Y}^{+}\left(t_{i}\right), i=\overline{1, N-1}$.

Справедлива следующая теорема относительно оценки величины $d\left(\widetilde{Y}^{-}\left(t_{i}\right), \widetilde{Y}^{+}\left(t_{i}\right)\right)$ рассогласования между нижними и верхними аппроксимациями $\widetilde{Y}^{-}\left(t_{i}\right), \widetilde{Y}^{+}\left(t_{i}\right), t_{i} \in \Gamma$ множеств достижимости $Y\left(t_{i}\right)$. 
Теорема. Пусть $X_{0} \in \operatorname{comp}\left(\mathbb{R}^{m}\right)$ - стартовое множество для системь (1.1), отвечающее моменту $t_{0}, \Gamma=\left\{t_{0}, t_{1}, \ldots, t_{i}, \ldots, t_{N}=\vartheta\right\}$ - разбиение промежутка $\left[t_{0}, \vartheta\right]$ с диаметром $\Delta=\underset{i=\overline{0, N-1}}{\max }\left(t_{i+1}-t_{i}\right)$.

Тогда нижние и верхние аппроксимации $\widetilde{Y}^{-}\left(t_{i}\right), \widetilde{Y}^{+}\left(t_{i}\right)$ множеств достижсмости $Y\left(t_{i}\right)$, $t_{i} \in \Gamma$, удовлетворяют соотношению

$$
d\left(\widetilde{Y}^{-}\left(t_{i}\right), \tilde{Y}^{+}\left(t_{i}\right)\right) \leq e^{L\left(t_{i}-t_{0}\right)}\left(\vartheta-t_{0}\right)\left(\gamma_{*}+1\right) \varphi(\Delta) ;
$$

здесь числа $L, \gamma_{*}$ и функиия $\varphi(\delta), \delta>0$, определены в разд. 1.

Д о к а з а т е л ь с т в о. Рассмотрим начальный промежуток $\left[t_{0}, t_{1}\right]$ разбиения $\Gamma$ и отвечающие его конечному моменту $t_{1}$ множества $Y\left(t_{1}\right), \widetilde{Y}^{-}\left(t_{1}\right), \widetilde{Y}^{+}\left(t_{1}\right)$. Принимая во внимание $(1.7)$, получаем $\widetilde{Y}^{-}\left(t_{1}\right) \subset Y\left(t_{1}\right) \subset \widetilde{Y}^{+}\left(t_{1}\right)$.

Оценим сверху расстояние $d\left(Y^{-}\left(t_{1}\right), Y^{+}\left(t_{1}\right)\right)$ между нижней и верхней аппроксимациями множества $Y\left(t_{1}\right)$.

Для этого сначала оценим сверху величину $d\left(\widetilde{Y}^{-}\left(t_{1}, t_{0}, x^{(0)}\right), \widetilde{Y}^{+}\left(t_{1}, t_{0}, x^{(0)}\right)\right)$ для каждой точки $x^{(0)} \in X_{0}$. Справедливо неравенство

$$
\begin{gathered}
d\left(\widetilde{Y}^{-}\left(t_{1}, t_{0}, x^{(0)}\right), \tilde{Y}^{+}\left(t_{1}, t_{0}, x^{(0)}\right)\right) \\
\leq d\left(\widetilde{Y}^{-}\left(t_{1}, t_{0}, x^{(0)}\right), \widetilde{Y}\left(t_{1}, t_{0}, x^{(0)}\right)\right)+d\left(\widetilde{Y}\left(t_{1}, t_{0}, x^{(0)}\right), \widetilde{Y}^{+}\left(t_{1}, t_{0}, x^{(0)}\right)\right) .
\end{gathered}
$$

Первое слагаемое в правой части этого неравенства представимо в виде

$$
\begin{gathered}
d\left(\widetilde{Y}^{-}\left(t_{1}, t_{0}, x^{(0)}\right), \tilde{Y}\left(t_{1}, t_{0}, x^{(0)}\right)\right)=d\left(x^{(0)}+\Delta F\left(t_{0}, x^{(0)}\right)_{-\varphi(\Delta)}, x^{(0)}\right. \\
\left.+\Delta F\left(t_{0}, x^{(0)}\right)\right)=\Delta d\left(F\left(t_{0}, x^{(0)}\right)_{-\varphi(\Delta)}, F\left(t_{0}, x^{(0)}\right)\right) .
\end{gathered}
$$

Принимая во внимание условие $\mathbf{C}$ и утверждение, сформулированное выше, получаем

$$
\Delta d\left(F\left(t_{0}, x^{(0)}\right)_{-\varphi(\Delta)}, F\left(t_{0}, x^{(0)}\right)\right) \leq \gamma_{*} \varphi(\Delta) .
$$

Следовательно, справедлива оценка

$$
d\left(\widetilde{Y}^{-}\left(t_{1}, t_{0}, x^{(0)}\right), \tilde{Y}\left(t_{1}, t_{0}, x^{(0)}\right)\right) \leq \gamma_{*} \omega(\Delta) .
$$

Также справедлива оценка

$$
d\left(\widetilde{Y}\left(t_{1}, t_{0}, x^{(0)}\right), \widetilde{Y}^{+}\left(t_{1}, t_{0}, x^{(0)}\right)\right) \leq \omega(\Delta) .
$$

Из (2.1) и (2.2) имеем

$$
d\left(\widetilde{Y}^{-}\left(t_{1}, t_{0}, x^{(0)}\right), \tilde{Y}^{+}\left(t_{1}, t_{0}, x^{(0)}\right)\right) \leq\left(\gamma_{*}+1\right) \omega(\Delta), \quad\left(t_{0}, x^{(0)}\right) \in \mathcal{D}, \quad x^{(0)} \in X_{0} .
$$

Из (2.3) получаем

$$
d\left(\widetilde{Y}^{-}\left(t_{1}\right), \widetilde{Y}^{+}\left(t_{1}\right)\right) \leq\left(\gamma_{*}+1\right) \omega\left(\Delta_{0}\right) .
$$

Рассмотрим следующий промежуток $\left[t_{1}, t_{2}\right]$ разбиения Г. Справедливы включения

$$
\begin{aligned}
\tilde{Y}^{-}\left(t_{2}\right) & =\widetilde{Y}^{-}\left(t_{2}, t_{1}, \tilde{Y}^{-}\left(t_{1}\right)\right) \subset \widetilde{Y}^{-}\left(t_{2}, t_{1}, Y\left(t_{1}\right)\right) \subset Y\left(t_{2}, t_{1}, Y\left(t_{1}\right)\right) \\
& \subset Y\left(t_{2}, t_{1}, \widetilde{Y}^{+}\left(t_{1}\right)\right) \subset \widetilde{Y}^{+}\left(t_{2}, t_{1}, \widetilde{Y}^{+}\left(t_{1}\right)\right)=\widetilde{Y}^{+}\left(t_{2}\right) .
\end{aligned}
$$

В итоге имеем включение $\widetilde{Y}^{-}\left(t_{2}\right) \subset Y\left(t_{2}\right) \subset \widetilde{Y}^{+}\left(t_{2}\right)$.

Оценим сверху рассогласование $d\left(\widetilde{Y}^{-}\left(t_{2}\right), \widetilde{Y}^{+}\left(t_{2}\right)\right)=h\left(\widetilde{Y}^{+}\left(t_{2}\right), \widetilde{Y}^{-}\left(t_{2}\right)\right)$ между нижней и верхней аппроксимациями множества $Y\left(t_{2}\right)$. Для этого выберем произвольную точку $x^{(1)} \in$ $\widetilde{Y}^{-}\left(t_{1}\right)$ и оценим сверху величину

$$
d\left(\widetilde{Y}^{-}\left(t_{2}, t_{1}, x^{(1)}\right), \tilde{Y}^{+}\left(t_{2}, t_{1}, x^{(1)}\right)\right)=h\left(\widetilde{Y}^{+}\left(t_{2}, t_{1}, x^{(1)}\right), \widetilde{Y}^{-}\left(t_{2}, t_{1}, x^{(1)}\right)\right) .
$$




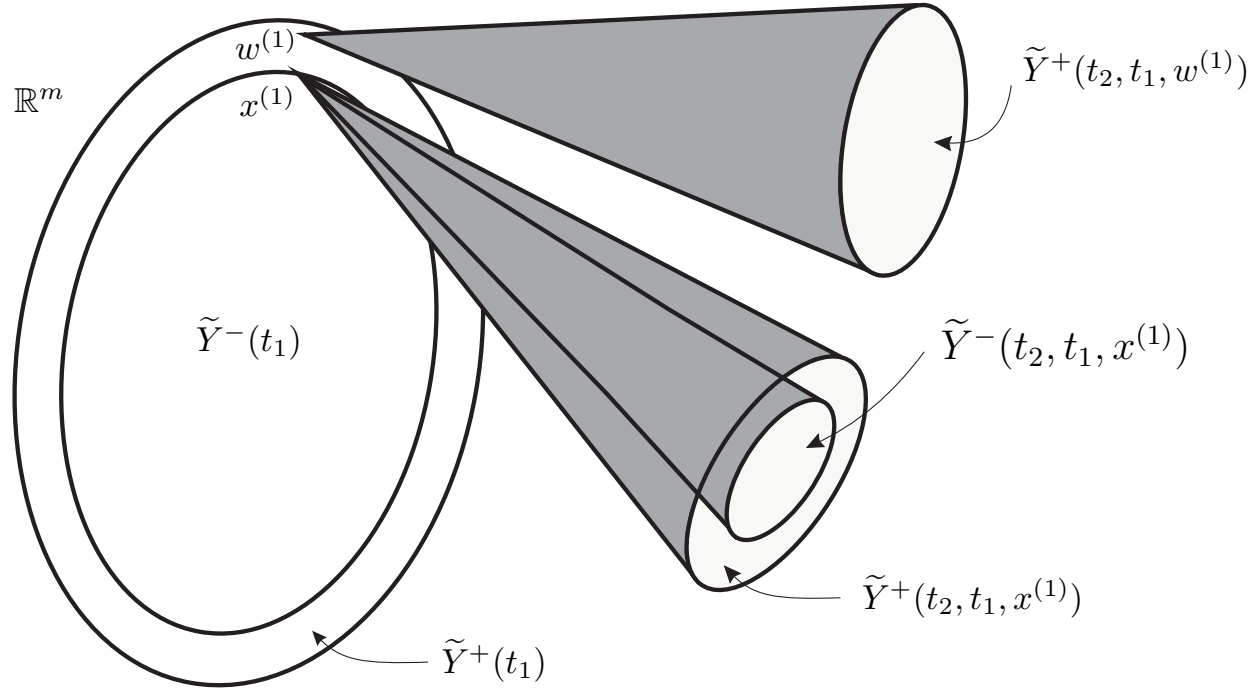

Нижняя и верхняя аппроксимации множества достижимости $\widetilde{Y}\left(t_{2}, t_{1}, x^{(1)}\right)$.

Для $x^{(1)}$ справедлива оценка, аналогичная (2.3): $d\left(\widetilde{Y}^{-}\left(t_{2}, t_{1}, x^{(1)}\right), \widetilde{Y}^{+}\left(t_{2}, t_{1}, x^{(1)}\right)\right) \leq$ $\left(\gamma_{*}+1\right) \omega(\Delta)$. Отсюда следует

$$
\begin{gathered}
d\left(\widetilde{Y}^{-}\left(t_{2}, t_{1}, \widetilde{Y}^{-}\left(t_{1}\right)\right), \widetilde{Y}^{+}\left(t_{2}, t_{1}, \widetilde{Y}^{-}\left(t_{1}\right)\right)\right)=\sup _{x^{(1)} \in \widetilde{Y}^{-}\left(t_{1}\right)} \inf _{w^{(1)} \in \widetilde{Y}^{-}\left(t_{1}\right)} h\left(\widetilde{Y}^{+}\left(t_{2}, t_{1}, x^{(1)}\right), \widetilde{Y}^{-}\left(t_{2}, t_{1}, w^{(1)}\right)\right) \\
\leq \sup _{x^{(1)} \in \widetilde{Y}^{-}\left(t_{1}\right)} h\left(\widetilde{Y}^{+}\left(t_{2}, t_{1}, x^{(1)}\right), \widetilde{Y}^{-}\left(t_{2}, t_{1}, x^{(1)}\right)\right) \leq\left(\gamma_{*}+1\right) \omega(\Delta) .
\end{gathered}
$$

Рассмотрим теперь произвольную точку $w^{(1)} \in \widetilde{Y}^{+}\left(t_{1}\right)$ и ближайшую в $\widetilde{Y}^{-}\left(t_{1}\right)$ к ней точку $x^{(1)}$ (см. рисунок). Для этих точек справедливо

$$
\begin{gathered}
d\left(\widetilde{Y}^{+}\left(t_{2}, t_{1}, w^{(1)}\right), \widetilde{Y}^{+}\left(t_{2}, t_{1}, x^{(1)}\right)\right) \leq e^{L \Delta_{1}}\left\|w^{(1)}-x^{(1)}\right\|=e^{L \Delta_{1}} \rho\left(w^{(1)}, \widetilde{Y}^{-}\left(t_{1}\right)\right) \\
\leq e^{L \Delta_{1}} h\left(\widetilde{Y}^{+}\left(t_{1}\right), \widetilde{Y}^{-}\left(t_{1}\right)\right)=e^{L \Delta_{1}} d\left(\widetilde{Y}^{-}\left(t_{1}\right), \widetilde{Y}^{+}\left(t_{1}\right)\right) .
\end{gathered}
$$

Имеет место оценка

$$
\begin{gathered}
d\left(\widetilde{Y}^{+}\left(t_{2}, t_{1}, w^{(1)}\right), \widetilde{Y}^{+}\left(t_{2}, t_{1}, \widetilde{Y}^{-}\left(t_{1}\right)\right)\right) \\
\leq d\left(\widetilde{Y}^{+}\left(t_{2}, t_{1}, w^{(1)}\right), \widetilde{Y}^{+}\left(t_{2}, t_{1}, x^{(1)}\right)\right) \leq e^{L \Delta_{1}} d\left(\widetilde{Y}^{-}\left(t_{1}\right), \widetilde{Y}^{+}\left(t_{1}\right)\right),
\end{gathered}
$$

из которой следует

$$
\begin{gathered}
d\left(\widetilde{Y}^{+}\left(t_{2}\right), \widetilde{Y}^{+}\left(t_{2}, t_{1}, \widetilde{Y}^{-}\left(t_{1}\right)\right)\right)=\sup _{w^{(1)} \in \widetilde{Y}^{+}\left(t_{1}\right)} d\left(\widetilde{Y}^{+}\left(t_{2}, t_{1}, w^{(1)}\right), \widetilde{Y}^{+}\left(t_{2}, t_{1}, \widetilde{Y}^{-}\left(t_{1}\right)\right)\right) \\
\leq e^{L \Delta_{1}} d\left(\widetilde{Y}^{-}\left(t_{1}\right), \widetilde{Y}^{+}\left(t_{1}\right)\right) .
\end{gathered}
$$

Отметим, что неравенство (2.5) может быть записано в виде

$$
d\left(\widetilde{Y}^{+}\left(t_{2}, t_{1}, \widetilde{Y}^{-}\left(t_{1}\right)\right), \tilde{Y}^{-}\left(t_{2}\right)\right) \leq\left(\gamma_{*}+1\right) \omega(\Delta) .
$$

Учитывая (2.6) и $(2.7)$, получаем

$$
d\left(\tilde{Y}^{-}\left(t_{2}\right), \tilde{Y}^{+}\left(t_{2}\right)\right) \leq e^{L \Delta_{1}} d\left(\tilde{Y}^{-}\left(t_{1}\right), \tilde{Y}^{+}\left(t_{1}\right)\right)+\left(\gamma_{*}+1\right) \omega\left(\Delta_{1}\right) .
$$

Продолжая последовательно перебирать промежутки $\left[t_{i}, t_{i+1}\right], i=2, \ldots, N-1$, и оценивать хаусдорфовы расстояния $d\left(\widetilde{Y}^{-}\left(t_{i}\right), \widetilde{Y}^{+}\left(t_{i}\right)\right)$ через предыдущие расстояния $d\left(\widetilde{Y}^{-}\left(t_{i-1}\right)\right.$, $\left.\tilde{Y}^{+}\left(t_{i-1}\right)\right)$, получаем оценки, аналогичные $(2.8)$ :

$$
d\left(\widetilde{Y}^{-}\left(t_{i}\right), \widetilde{Y}^{+}\left(t_{i}\right)\right) \leq e^{L \Delta_{i-1}} d\left(\widetilde{Y}^{-}\left(t_{i-1}\right), \tilde{Y}^{+}\left(t_{i-1}\right)\right)+\left(\gamma_{*}+1\right) \omega\left(\Delta_{i-1}\right), \quad i=\overline{2, N-1} .
$$


Из оценок (2.4) и (2.9) выводим оценку

$$
\begin{gathered}
d\left(\widetilde{Y}^{-}\left(t_{i}\right), \widetilde{Y}^{+}\left(t_{i}\right)\right) \leq e^{L\left(t_{i}-t_{0}\right)} \sum_{k=\overline{1, i-1}}\left(\gamma_{*}+1\right) \omega\left(\Delta_{k}\right) \\
=e^{L\left(t_{i}-t_{0}\right)}\left(t_{i}-t_{0}\right)\left(\gamma_{*}+1\right) \varphi(\Delta), \quad i=\overline{2, N-1} .
\end{gathered}
$$

Огрубляя оценки (2.10), получаем

$$
d\left(\widetilde{Y}^{-}\left(t_{i}\right), \widetilde{Y}^{+}\left(t_{i}\right)\right) \leq e^{L\left(t_{i}-t_{0}\right)}\left(\theta-t_{0}\right)\left(\gamma_{*}+1\right) \varphi(\Delta) .
$$

Из (2.11) вытекает предельное соотношение

$$
\lim _{\Delta=\Delta(\Gamma) \downarrow 0} \max _{i=\overline{0, N}} d\left(\tilde{Y}^{-}\left(t_{i}\right), \tilde{Y}^{+}\left(t_{i}\right)\right)=0 .
$$

Теорема доказана.

\section{3. К вычислению характеристик, участвующих в оценке множеств достижимости некоторых управляемых систем}

В этом разделе рассматриваются конкретные нелинейные по $(t, x)$ и линейные по $u$ управляемые системы в пространстве $\mathbb{R}^{2}$ и показывается, как для этих систем можно вычислить некоторые характеристические элементы (числовые величины и функции), присутствующие в нижних и верхних оценках множеств достижимости этих систем.

На промежутке времени $\left[t_{0}, \theta\right], t_{0}<\theta<\infty$, рассмотрим управляемую систему

$$
\begin{gathered}
\frac{d x}{d t}=s(t, x)+B(t, x) u, \\
u=u(t) \in P, \\
(t, x) \in\left[t_{0}, \theta\right] \times \mathbb{R}^{2} ;
\end{gathered}
$$

здесь $t$ - время, $x=\left(\begin{array}{l}x_{1} \\ x_{2}\end{array}\right)$ - фазовый вектор системы из $\mathbb{R}^{2}, u$ - вектор управления, $P-$ выпуклый компакт в $\mathbb{R}^{2}$.

Предполагаем, что система (3.1) удовлетворяет следующим условиям.

А. Вектор-функция $s(t, x)$ ограничена и непрерывно-дифференцируема на $\left[t_{0}, \theta\right] \times \mathbb{R}^{2}$.

В. Матрица-функция $B(t, x)=\left(b_{i j}(t, x)\right)(i, j=\overline{1,2})$ с нормой

$$
\|B(t, x)\|=\left(\sum_{i, j=\overline{1,2}} b_{i j}^{2}(t, x)\right)^{1 / 2}
$$

обладает непрерывно-дифференцируемыми коэффициентами $b_{i j}(t, x)$ на $\left[t_{0}, \theta\right] \times \mathbb{R}^{2}$, и при этом имеет место $\min _{(t, x) \in D}|\operatorname{det}(B(t, x))|>0$ на любом компакте $D$ из $\left[t_{0}, \theta\right] \times \mathbb{R}^{2}$.

Из $\mathbf{A}, \mathbf{B}$ следует, что правая часть системы (3.1) - вектор-функция $f(t, x, u)=s(t, x)+$ $B(t, x) u$ - удовлетворяет условиям

a)

$$
\begin{gathered}
K=K_{D}=\max \{\|f(t, x, u)\|:(t, x, u) \in D \times P\} \\
\leq \max \left\{\|s(t, x)\|+\|B(t, x)\| h_{P}:(t, x) \in D\right\} \leq K_{D}^{s}+K_{D}^{B} h_{P},
\end{gathered}
$$

где

$$
K_{D}^{s}=\max \{\|s(t, x)\|:(t, x) \in D\} \in(0, \infty),
$$




$$
K_{D}^{B}=\max \{\|B(t, x)\|:(t, x) \in D\} \in(0, \infty), \quad h_{P}=\max _{u \in P}\|u\|
$$

b)

$$
\left\|f\left(t, x^{(1)}\right)-f\left(t, x^{(2)}\right)\right\| \leq L\left\|x^{(1)}-x^{(2)}\right\|, \quad\left(t, x^{(1)}\right),\left(t, x^{(2)}\right) \in D, \quad u \in P .
$$

Здесь постоянная $L \in(0, \infty)$ определена равенством $L=L^{s}+L^{B} h_{P}, L^{s}$ и $L^{B}$ - постоянные Липшица по переменной $x$ на компакте $D$ соответственно вектор-функции $s(t, x)$ и матрицыфункции $B(t, x)$, причем $L^{B}=\left(\sum_{i, j=\overline{1,2}} k_{i j}^{2}\right)^{1 / 2}$ числа $k_{i j} \in(0, \infty)$ - постоянные Липшица по $x$ скалярных функций $b_{i j}(t, x)$ на $D$.

Еще один важный характеристический элемент в наших оценках множеств достижимости - модуль непрерывности по $(t, x)$ правой части $f(t, x, u)$ системы $(3.1)$ - вычисляется по формуле

$$
\omega_{D}^{*}(\delta)=\max \left\{\left\|f\left(t_{*}, x_{*}, u\right)-f\left(t^{*}, x^{*}, u\right)\right\|:\left|t^{\prime}-t^{\prime \prime}\right|+\left\|x_{*}-x^{*}\right\| \leq \delta,\left(t_{*}, x_{*}\right),\left(t^{*}, x^{*}\right) \in D, u \in P\right\} .
$$

Рассмотрим также выпуклые компакты

$$
F(t, x)=\{f(t, x, u): u \in P\}=\{s(t, x)+B(t, x) u: u \in P\}
$$

в пространстве $\mathbb{R}^{2}$, удовлетворяющие следующему условию.

C. Множества $F(t, x),(t, x) \in D$, обладают свойствами

$\mathrm{a}^{*}$ ) множества $F(t, x),(t, x) \in D$, равноограничены снизу в том смысле, что

$$
\exists \rho \in(0, \infty): \forall(t, x) \in D \exists B(z(t, x) ; \rho) \subset F(t, x) ;
$$

$\mathrm{b}^{*}$ ) коэффициенты вытянутости $\gamma_{F(t, x)},(t, x) \in D$ равноограничены сверху в том смысле, что

$$
\exists \gamma \in(0, \infty): \sup _{(t, x) \in D} \gamma_{F(t, x)} \leq \gamma
$$

Здесь $\gamma_{F(t, x)}=\frac{d_{F(t, x)}}{r_{F(t, x)}}, d_{F(t, x)}$ и $r_{F(t, x)}$ соответственно диаметр множества $F(t, x)$ и радиус вписанного шара в $F(t, x)$.

Приступим теперь к вычислению характеристических элементов правой части управляемой системы (3.1) в зависимости от вида компакта $P$ из (3.2). Рассмотрим два варианта ограничений на $P$.

Вариант 1. Пусть $u=u(t) \in P=\left\{\left(u_{1}, u_{2}\right):\left|u_{1}\right| \leq 1,\left|u_{2}\right| \leq 1\right\}$ - единичный квадрат в плоскости $\mathbb{R}^{2}$ с центром в точке $O=\left(\begin{array}{l}0 \\ 0\end{array}\right)$.

Обозначим вершины квадрата $P$ через $A=\left(\begin{array}{l}1 \\ 1\end{array}\right), B=\left(\begin{array}{c}-1 \\ 1\end{array}\right), C=\left(\begin{array}{c}-1 \\ -1\end{array}\right), D=\left(\begin{array}{c}1 \\ -1\end{array}\right)$. При любом фиксированном $\left(t^{*}, x^{*}\right) \in D$ отображение $u \mapsto B\left(t^{*}, x^{*}\right) u$ линейно по $u$, откуда следует, что множество $G_{\left(t^{*}, x^{*}\right)}=\left\{B\left(t^{*}, x^{*}\right) u: u \in P\right\}-$ параллелограмм в $\mathbb{R}^{2}$. Справедливы равенства $B\left(t^{*}, x^{*}\right) \cdot 0=\left(\begin{array}{l}0 \\ 0\end{array}\right)$ и $B\left(t^{*}, x^{*}\right) \cdot(-u)=-B\left(t^{*}, x^{*}\right) u$.

Пусть точки $A^{*}, B^{*}, C^{*}, D^{*}$ определяются соотношениями

$$
\begin{gathered}
A^{*}=B\left(t^{*}, x^{*}\right) A=\left(\begin{array}{c}
b_{11}\left(t^{*}, x^{*}\right)+b_{12}\left(t^{*}, x^{*}\right) \\
b_{21}\left(t^{*}, x^{*}\right)+b_{22}\left(t^{*}, x^{*}\right)
\end{array}\right) ; \\
B^{*}=B\left(t^{*}, x^{*}\right) B=\left(\begin{array}{l}
-b_{11}\left(t^{*}, x^{*}\right)+b_{12}\left(t^{*}, x^{*}\right) \\
-b_{21}\left(t^{*}, x^{*}\right)+b_{22}\left(t^{*}, x^{*}\right)
\end{array}\right) ;
\end{gathered}
$$




$$
C^{*}=-A^{*}=\left(\begin{array}{c}
-b_{11}\left(t^{*}, x^{*}\right)-b_{12}\left(t^{*}, x^{*}\right) \\
-b_{21}\left(t^{*}, x^{*}\right)-b_{22}\left(t^{*}, x^{*}\right)
\end{array}\right) ; \quad D^{*}=-B^{*}=\left(\begin{array}{c}
b_{11}\left(t^{*}, x^{*}\right)-b_{12}\left(t^{*}, x^{*}\right) \\
b_{21}\left(t^{*}, x^{*}\right)-b_{22}\left(t^{*}, x^{*}\right)
\end{array}\right) .
$$

Диаметр множества $F\left(t^{*}, x^{*}\right)$ равен диаметру множества $G\left(t^{*}, x^{*}\right)$, при этом диаметр параллелограмма $G\left(t^{*}, x^{*}\right)$ равен длине максимальной из его диагоналей, т. е.

$$
d_{G\left(t^{*}, x^{*}\right)}=2 \max \left\{\left\|\left(\begin{array}{c}
b_{11}\left(t^{*}, x^{*}\right)+b_{12}\left(t^{*}, x^{*}\right) \\
b_{21}\left(t^{*}, x^{*}\right)+b_{22}\left(t^{*}, x^{*}\right)
\end{array}\right)\right\|,\left\|\left(\begin{array}{c}
b_{11}\left(t^{*}, x^{*}\right)-b_{12}\left(t^{*}, x^{*}\right) \\
b_{21}\left(t^{*}, x^{*}\right)-b_{22}\left(t^{*}, x^{*}\right)
\end{array}\right)\right\|\right\} .
$$

Уравнения прямых $\Pi_{A^{*}, B^{*}}$ и $\Pi_{A^{*}, D^{*}}$ в плоскости $\mathbb{R}^{2}$, проходящих соответственно через пары точек $A^{*}, B^{*}$ и $A^{*}, D^{*}$, имеют вид

$$
\begin{aligned}
& \Pi_{A^{*}, B^{*}}: b_{21}\left(t^{*}, x^{*}\right) u_{1}-b_{11}\left(t^{*}, x^{*}\right) u_{2}+\left|\operatorname{det}\left(B\left(t^{*}, x^{*}\right)\right)\right|=0 ; \\
& \Pi_{A^{*}, D^{*}}: b_{22}\left(t^{*}, x^{*}\right) u_{1}-b_{12}\left(t^{*}, x^{*}\right) u_{2}-\left|\operatorname{det}\left(B\left(t^{*}, x^{*}\right)\right)\right|=0 .
\end{aligned}
$$

Из равенств (3.4), (3.5) определяем расстояния от точки $O$ до прямых $\Pi_{A^{*}, B^{*}}$ и $\Pi_{A^{*}, D^{*}}$ :

$$
\rho\left(O ; \Pi_{A^{*}, B^{*}}\right)=\frac{\left|\operatorname{det}\left(B\left(t^{*}, x^{*}\right)\right)\right|}{\sqrt{b_{11}^{2}\left(t^{*}, x^{*}\right)+b_{21}^{2}\left(t^{*}, x^{*}\right)}} ; \quad \rho\left(O ; \Pi_{A^{*}, D^{*}}\right)=\frac{\left|\operatorname{det}\left(B\left(t^{*}, x^{*}\right)\right)\right|}{\sqrt{b_{12}^{2}\left(t^{*}, x^{*}\right)+b_{22}^{2}\left(t^{*}, x^{*}\right)}} .
$$

Справедливо равенство для радиусов кругов, вписанных в множества $F\left(t^{*}, x^{*}\right)$ и $G\left(t^{*}, x^{*}\right)$ :

$$
r_{\left(t^{*}, x^{*}\right)}=r_{G\left(t^{*}, x^{*}\right)}=\min \left\{\rho\left(O ; \Pi_{A^{*}, B^{*}}\right), \rho\left(O ; \Pi_{A^{*}, D^{*}}\right)\right\}
$$

Тогда коэффициент вытянутости $\gamma_{F\left(t^{*}, x^{*}\right)}$ множества $F\left(t^{*}, x^{*}\right)$ удовлетворяет соотношениям

$$
\begin{gathered}
\gamma_{F\left(t^{*}, x^{*}\right)}=\gamma_{G\left(t^{*}, x^{*}\right)}, \\
\gamma_{F\left(t^{*}, x^{*}\right)} \leq d_{G\left(t^{*}, x^{*}\right)} \frac{\left(\sum_{i, j=\overline{1,2}} b_{i j}^{2}(t, x)\right)^{1 / 2}}{\left|\operatorname{det}\left(B\left(t^{*}, x^{*}\right)\right)\right|}=d_{G\left(t^{*}, x^{*}\right)} \frac{\left\|B\left(t^{*}, x^{*}\right)\right\|}{\left|\operatorname{det}\left(B\left(t^{*}, x^{*}\right)\right)\right|} \leq \frac{2 \sqrt{2}\left\|B\left(t^{*}, x^{*}\right)\right\|^{2}}{\left|\operatorname{det}\left(B\left(t^{*}, x^{*}\right)\right)\right|} .
\end{gathered}
$$

Тем самым задача о вычислении константы, ограничивающей сверху коэффициенты вытянутости множеств $F(t, x)$ на компакте $D \subset\left[t_{0}, \theta\right] \times \mathbb{R}^{2}$, свелась к задаче о вычислении максимума функции $\frac{\|B(t, x)\|^{2}}{|\operatorname{det}(B(t, x))|}$ на компакте $D \subset\left[t_{0}, \theta\right] \times \mathbb{R}^{2}$.

Вариант 2. Пусть $P=\left\{u=\left(u_{1}, u_{2}\right): u_{1}^{2}+u_{2}^{2} \leq 1\right\}-$ замкнутый круг в $\mathbb{R}^{2}$ с центром в точке $O=\left(\begin{array}{l}0 \\ 0\end{array}\right)$.

При любой фиксированной точке $\left(t^{*}, x^{*}\right) \in D$ отображение $u \mapsto B\left(t^{*}, x^{*}\right) u$ линейно по $u$, откуда следует, что множество $G_{\left(t^{*}, x^{*}\right)}=\left\{B\left(t^{*}, x^{*}\right) u: u \in P\right\}$ - эллипс в $\mathbb{R}^{2}$. Как и в предыдущем примере, $B\left(t^{*}, x^{*}\right)(O)=\left(\begin{array}{l}0 \\ 0\end{array}\right)$ и $B\left(t^{*}, x^{*}\right)(-u)=-B\left(t^{*}, x^{*}\right) u$.

Радиус вписанного в $G_{\left(t^{*}, x^{*}\right)}$ шара и диаметр эллипса $G_{\left(t^{*}, x^{*}\right)}$ соответственно равны длинам малой полуоси и большой оси эллипса. Иными словами, для их вычисления требуется найти

$$
\min _{u \in P}\left\|B\left(t^{*}, x^{*}\right) u\right\| \quad \text { и } \max _{u \in P}\left\|B\left(t^{*}, x^{*}\right) u\right\| .
$$

Очевидно, что указанные выше экстремумы функции $H(u)=\left\|B\left(t^{*}, x^{*}\right) u\right\|$ достигаются на границе шара $P$, т.е. на сфере $S=\left\{u=\left(u_{1}, u_{2}\right): u_{1}^{2}+u_{2}^{2}=1\right\}$. Также в тех же точках на сфере $S$ достигаются экстремумы функции $H^{2}(u)$ на $P$. В силу осесимметричности эллипса достаточно искать экстремумы функции $H(u)$ при $u_{1} \geq 0$. 
Введем функцию Лагранжа $L\left(u_{1}, u_{2}, \lambda\right)=H^{2}\left(u_{1}, u_{2}\right)+\lambda\left(u_{1}^{2}+u_{2}^{2}-1\right)$. Необходимые условия для нахождения точек экстремума в нашей задаче выглядят так:

$$
\left\{\begin{array}{c}
2 b_{11}\left(b_{11} u_{1}+b_{12} u_{2}\right)+2 b_{21}\left(b_{21} u_{1}+b_{22} u_{2}\right)+2 \lambda u_{1}=0 \\
2 b_{12}\left(b_{11} u_{1}+b_{12} u_{2}\right)+2 b_{22}\left(b_{21} u_{1}+b_{22} u_{2}\right)+2 \lambda u_{2}=0 \\
\lambda\left(u_{1}^{2}+u_{2}^{2}-1\right)=0 \\
\lambda \neq 0
\end{array}\right.
$$

Перепишем систему (3.7) в виде

$$
\left\{\begin{array}{c}
b_{11}\left(b_{11} u_{1}+b_{12} u_{2}\right)+b_{21}\left(b_{21} u_{1}+b_{22} u_{2}\right)=-\lambda u_{1} \\
b_{12}\left(b_{11} u_{1}+b_{12} u_{2}\right)+b_{22}\left(b_{21} u_{1}+b_{22} u_{2}\right)=-\lambda u_{2} \\
u_{1}^{2}+u_{2}^{2}=1
\end{array}\right.
$$

Из (3.8) следует, что любая точка упомянутого экстремума $u=\left(u_{1}, u_{2}\right)$ отлична от $(0,0)$. Перемножив первые два уравнения системы (3.8), получим равенство

$$
-\lambda u_{2}\left(b_{11}\left(b_{11} u_{1}+b_{12} u_{2}\right)+b_{21}\left(b_{21} u_{1}+b_{22} u_{2}\right)\right)=-\lambda u_{1}\left(b_{12}\left(b_{11} u_{1}+b_{12} u_{2}\right)+b_{22}\left(b_{21} u_{1}+b_{22} u_{2}\right)\right) .
$$

Поделив обе части уравнения (3.9) на $\lambda$ и приведя подобные, получим

$$
\left(b_{11} b_{12}+b_{21} b_{22}\right) u_{1}^{2}+\left(b_{11}^{2}+b_{21}^{2}-b_{12}^{2}-b_{22}^{2}\right) u_{1} u_{2}-\left(b_{11} b_{12}+b_{21} b_{22}\right) u_{2}^{2}=0 .
$$

Введем для упрощения обозначения $a_{1}=b_{11} b_{12}+b_{21} b_{22}, a_{2}=b_{11}^{2}+b_{21}^{2}-b_{12}^{2}-b_{22}^{2}$, в которых уравнение (3.10) запишется в виде

$$
a_{1}\left(u_{1}^{2}-u_{2}^{2}\right)+a_{2} u_{1} u_{2}=0 .
$$

Сначала рассмотрим случай $a_{1} \neq 0$. Тогда из (3.11) следует, что $u_{2} \neq 0$. Поделив обе части равенства (3.11) на $u_{2}^{2}$ и введя замену $v=u_{1} / u_{2}$, имеем

$$
a_{1} v^{2}+a_{2} v-1-a_{1}=0
$$

откуда

$$
v_{1}=\frac{-a_{2}+\sqrt{4 a_{1}^{2}+a_{2}^{2}}}{2 a_{1}}, \quad v_{2}=\frac{-a_{2}-\sqrt{4 a_{1}^{2}+a_{2}^{2}}}{2 a_{1}} .
$$

Из обратной замены и условия $u_{1}^{2}+u_{2}^{2}=1$ определяем точки экстремума

$$
U_{1}=\left(\begin{array}{l}
u_{1} \\
u_{2}
\end{array}\right)=\left(\begin{array}{c}
\frac{v_{1}}{\sqrt{v_{1}^{2}+1}} \\
\frac{1}{\sqrt{v_{1}^{2}+1}}
\end{array}\right), \quad U_{2}=\left(\begin{array}{l}
u_{1} \\
u_{2}
\end{array}\right)=\left(\begin{array}{c}
-\frac{v_{2}}{\sqrt{v_{2}^{2}+1}} \\
-\frac{1}{\sqrt{v_{2}^{2}+1}}
\end{array}\right) .
$$

Непосредственной подстановкой получаем

$$
H^{2}\left(U_{1}\right)=\frac{1}{2}\|B\|^{2}+\frac{\sqrt{4 a_{1}^{2}+a_{2}^{2}}}{2}, \quad H^{2}\left(U_{2}\right)=\frac{1}{2}\|B\|^{2}-\frac{\sqrt{4 a_{1}^{2}+a_{2}^{2}}}{2},
$$

из чего следует, что

$$
\begin{gathered}
r_{G\left(t^{*}, x^{*}\right)}=\min _{u \in P} H(u)=\sqrt{\frac{1}{2}\|B\|^{2}-\frac{\sqrt{4 a_{1}^{2}+a_{2}^{2}}}{2}} ; \\
d_{G\left(t^{*}, x^{*}\right)}=2 \max _{u \in P} H(u)=2 \sqrt{\frac{1}{2}\|B\|^{2}+\frac{\sqrt{4 a_{1}^{2}+a_{2}^{2}}}{2}} ;
\end{gathered}
$$




$$
\gamma_{G\left(t^{*}, x^{*}\right)}=\frac{d_{G\left(t^{*}, x^{*}\right)}}{r_{G\left(t^{*}, x^{*}\right)}}=2 \sqrt{\frac{\|B\|^{2}+\sqrt{4 a_{1}^{2}+a_{2}^{2}}}{\|B\|^{2}-\sqrt{4 a_{1}^{2}+a_{2}^{2}}}}
$$

в случае $a_{1} \neq 0$.

Теперь рассмотрим случай $a_{1}=0$. Тогда система (3.8) запишется в виде

$$
\left\{\begin{array}{c}
\left(b_{11}^{2}+b_{21}^{2}\right) u_{1}=-\lambda u_{1} \\
\left(b_{12}^{2}+b_{22}^{2}\right) u_{2}=-\lambda u_{2} \\
u_{1}^{2}+u_{2}^{2}=1
\end{array}\right.
$$

Если $a_{2} \neq 0$, то $b_{11}^{2}+b_{21}^{2} \neq b_{12}^{2}+b_{22}^{2}$. Поскольку хотя бы одно из выражений $b_{11}^{2}+b_{21}^{2}, b_{12}^{2}+b_{22}^{2}$ не равно $\lambda$, то либо $u_{1}=0$, либо $u_{2}=0$.

Точками экстремума в этом случае будут

$$
U_{1}=\left(\begin{array}{l}
u_{1} \\
u_{2}
\end{array}\right)=\left(\begin{array}{l}
1 \\
0
\end{array}\right), \quad U_{2}=\left(\begin{array}{l}
u_{1} \\
u_{2}
\end{array}\right)=\left(\begin{array}{l}
0 \\
1
\end{array}\right),
$$

откуда

$$
H^{2}\left(U_{1}\right)=b_{11}^{2}+b_{21}^{2}, \quad H^{2}\left(U_{2}\right)=b_{12}^{2}+b_{22}^{2} .
$$

Отметим, что здесь формулы для вычисления величин $r_{G\left(t^{*}, x^{*}\right)}, d_{G\left(t^{*}, x^{*}\right)}, \gamma_{G\left(t^{*}, x^{*}\right)}$ будут такими же, как и при $a_{1} \neq 0$.

В случае $a_{1}=a_{2}=0$ множество $G\left(t^{*}, x^{*}\right)$ превратится в круг, и формулы (3.12)-(3.14) останутся верными, причем в этом случае $\gamma_{G\left(t^{*}, x^{*}\right)}=2$.

Остается упомянуть, что аналогично предыдущему примеру $r_{F\left(t^{*}, x^{*}\right)}=r_{G\left(t^{*}, x^{*}\right)}, d_{F\left(t^{*}, x^{*}\right)}=$ $d_{G\left(t^{*}, x^{*}\right)}, \gamma_{F\left(t^{*}, x^{*}\right)}=\gamma_{G\left(t^{*}, x^{*}\right)}$, а исходная задача свелась к нахождению максимума функции $\gamma_{F(t, x)}$ на компакте $D$, явно выраженной через переменные $t, x$.

Теперь перейдем к рассмотрению управляемых систем с конкретной матрицей $B(t, x)$.

П р и м е р. Пусть на множестве $D=[3 ; 4] \times\left[\frac{\pi}{6} ; \frac{\pi}{4}\right]^{2}$ определена управляемая система

$$
\begin{gathered}
\frac{d x}{d t}=f(t, x)+B(t, x) u ; \\
u=u(t) \in P ; \\
(t, x) \in D \subset \mathbb{R}^{3},
\end{gathered}
$$

в которой $x=\left(\begin{array}{l}x_{1} \\ x_{2}\end{array}\right)$ - фазовый вектор системы, $B(t, x)=\left(\begin{array}{cc}\sqrt{2} \sin \left(t+x_{1}\right) & \sin t \\ \sqrt{2} \cos \left(t+x_{1}\right) & \cos t\end{array}\right), f(t, x)=0$.

Очевидно, что функция $f(t, x)$ удовлетворяет условию $\mathbf{A}$.

Вычислим определитель матрицы $B=B(t, x)$.

$$
\operatorname{det}(B)=\sqrt{2} \sin \left(t+x_{1}\right) \cos t-\sqrt{2} \cos \left(t+x_{1}\right) \sin t=\sqrt{2} \sin x_{1} .
$$

При $x_{1} \in\left[\frac{\pi}{6} ; \frac{\pi}{4}\right]$ величина $\sin x_{1}$ принимает значения из отрезка $\left[\frac{1}{2} ; \frac{\sqrt{2}}{2}\right]$, поэтому $\operatorname{det}(B)>0$, из чего следует выполнение условия $\mathbf{B}$.

Рассмотрим случай, когда $u=u(t) \in P=\left\{\left(u_{1}, u_{2}\right):\left|u_{1}\right| \leq 1,\left|u_{2}\right| \leq 1\right\}-$ единичный квадрат с центром в точке $O=\left(\begin{array}{l}0 \\ 0\end{array}\right)$.

Проверим выполнение условия С. Из формулы (3.6) следует

$$
\begin{aligned}
r_{F(t, x)} & =\min \left\{\frac{|\operatorname{det}(B(t, x))|}{\sqrt{b_{11}^{2}(t, x)+b_{21}^{2}(t, x)}}, \frac{|\operatorname{det}(B(t, x))|}{\sqrt{b_{12}^{2}(t, x)+b_{22}^{2}(t, x)}}\right\} \\
& =\min \left\{\frac{\sqrt{2}}{2 \sqrt{2 \sin ^{2}\left(t+x_{1}\right)+2 \cos ^{2}\left(t+x_{1}\right)}}, \frac{\sqrt{2}}{2 \sqrt{\sin ^{2} t+\cos ^{2} t}}\right\}=\frac{1}{2} .
\end{aligned}
$$


В результате видно, что коэффициент вытянутости $\gamma_{F(t, x)}$ зависит лишь от диаметра $d_{F(t, x)}$; в силу формулы (3.3) имеем

$$
\begin{aligned}
\gamma_{F(t, x)}=2 d_{F(t, x)} & =4 \max \left\{\left\|\left(\begin{array}{c}
b_{11}(t, x)+b_{12}(t, x) \\
b_{21}(t, x)+b_{22}(t, x)
\end{array}\right)\right\|,\left\|\left(\begin{array}{c}
b_{11}(t, x)-b_{12}(t, x) \\
b_{21}(t, x)-b_{22}(t, x)
\end{array}\right)\right\|\right\} \\
& =4 \max \left\{\left\|\left(\begin{array}{c}
\sqrt{2} \sin \left(t+x_{1}\right)+\sin (t) \\
\sqrt{2} \cos \left(t+x_{1}\right)+\cos (t)
\end{array}\right)\right\|,\left\|\left(\begin{array}{c}
\sqrt{2} \sin \left(t+x_{1}\right)-\sin (t) \\
\sqrt{2} \cos \left(t+x_{1}\right)-\cos (t)
\end{array}\right)\right\|\right\} \\
& =4 \max \left\{\sqrt{3+2 \sqrt{2} \cos x_{1}}, \sqrt{3-2 \sqrt{2} \cos x_{1}}\right\} .
\end{aligned}
$$

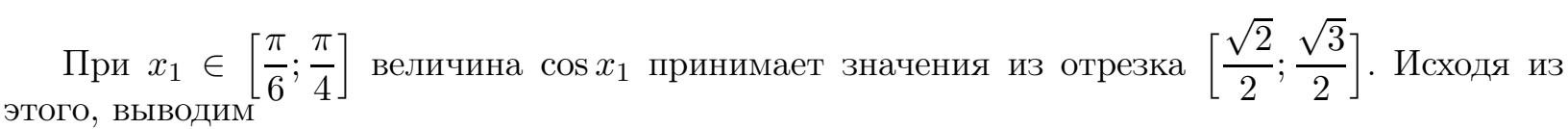

$$
\begin{gathered}
\max \sqrt{3+2 \sqrt{2} \cos x_{1}}=\sqrt{3+2 \sqrt{2} \cdot \frac{\sqrt{3}}{2}}=\sqrt{3+\sqrt{6}} \\
\max \sqrt{3-2 \sqrt{2} \cos x_{1}}=\sqrt{3-2 \sqrt{2} \cdot \frac{\sqrt{2}}{2}}=1 .
\end{gathered}
$$

В итоге $\gamma_{F(t, x)}=4 \sqrt{3+\sqrt{6}}$.

Теперь рассмотрим случай $u=u(t) \in P=\left\{\left(u_{1}, u_{2}\right) u_{1}^{2}+u_{2}^{2} \leq 1\right\}$ - замкнутый шар с центром в точке $O=\left(\begin{array}{l}0 \\ 0\end{array}\right)$.

Проверим выполнение условия С. Для этого вычислим норму матрицы $B$.

$$
\|B\|=\sqrt{2 \sin ^{2}\left(t+x_{1}\right)+2 \cos ^{2}\left(t+x_{1}\right)+\sin ^{2} t+\cos ^{2} t}=\sqrt{3} .
$$

Используя обозначения из варианта 2, получаем

$$
\begin{gathered}
a_{1}=\sqrt{2} \sin \left(t+x_{1}\right) \sin t+\sqrt{2} \cos \left(t+x_{1}\right) \cos t=\sqrt{2} \cos x_{1}, \\
a_{2}=2 \sin ^{2}\left(t+x_{1}\right)+2 \cos ^{2}\left(t+x_{1}\right)-\sin ^{2} t-\cos ^{2} t=1 .
\end{gathered}
$$

Из рассмотрения этого же примера известно, что

$$
r_{F(t, x)}=\sqrt{\frac{1}{2}\|B\|^{2}-\frac{\sqrt{4 a_{1}^{2}+a_{2}^{2}}}{2}}=\sqrt{\frac{3}{2}-\frac{\sqrt{8 \cos ^{2} x_{1}+1}}{2}} .
$$

Равенство (3.15) обращается в ноль только в случае $\cos ^{2} x_{1}=1$. Как было показано ранее, при $x_{1} \in\left[\frac{\pi}{6} ; \frac{\pi}{4}\right]$ такого быть не может.

Следовательно, $r_{F(t, x)} \neq 0$ на компакте $D$. Однако, на этом же компакте функция $r_{F(t, x)}$ достигает своего минимального значения $\rho>0$, а значит выполняется условие С. $a^{*}$ ).

Условие $\left.\mathbf{C} . b^{*}\right)$ выполнено в силу ограниченности множества $D$.

Теперь вычислим коэффициент вытянутости $\gamma_{F(t, x)}$. Из варианта 2 имеем

$$
\gamma_{F(t, x)}=2 \sqrt{\frac{\|B\|^{2}+\sqrt{4 a_{1}^{2}+a_{2}^{2}}}{\|B\|^{2}-\sqrt{4 a_{1}^{2}+a_{2}^{2}}}}=2 \sqrt{\frac{3+\sqrt{4 a_{1}^{2}+1}}{3-\sqrt{4 a_{1}^{2}+1}}}=2 \sqrt{\frac{6}{3-\sqrt{4 a_{1}^{2}+1}}-1},
$$

из чего следует, что максимум $\gamma_{F(t, x)}$ достигается при максимуме $a_{1}^{2}$.

Максимальное значение $a_{1}^{2}(t, x)=2 \cos ^{2} x_{1}$ на отрезке $\left[\frac{\pi}{6} ; \frac{\pi}{4}\right]$ достигается при $x_{1}=\frac{\pi}{4}$, откуда $\max _{(t, x) \in D} a_{1}^{2}(t, x)=1$. 
Подставляя максимальное значение $a_{1}^{2}$ в равенство (3.16), получаем

$$
\gamma_{F(t, x)}=2 \sqrt{\frac{6}{3-\sqrt{5}}-1}=3+\sqrt{5}
$$

Приступим к вычислению константы Липшица правой части системы (3.1). Для нашего примера условие липшицевости будет выглядеть следующим образом:

$$
\left\|B\left(t, x^{(1)}\right) u-B\left(t, x^{(2)}\right) u\right\| \leq K\|u\| \cdot\left\|x^{(1)}-x^{(2)}\right\|=K d_{P}\left\|x^{(1)}-x^{(2)}\right\|,
$$

где $d_{P}$ - диаметр множества $P$.

Найдем константы Липшица коэффициентов матрицы $B$. Функции $\sin t, \cos t$ не зависят от $x$, поэтому их константы липшицевости равны нулю.

Функций $\sqrt{2} \sin \left(t+x_{1}\right), \sqrt{2} \cos \left(t+x_{1}\right)$ дифференцируемы по $x_{1}$, значит их константы Липшица вычисляются как супремум модуля их производной по $x_{1}$ на множестве $D$.

При $\left(t, x_{1}\right) \in[3 ; 4] \times\left[\frac{\pi}{6} ; \frac{\pi}{4}\right]$ аргумент $t+x_{1}$ принимает значения из отрезка $\left[3+\frac{\pi}{6} ; 4+\frac{\pi}{4}\right]$, причем $3+\frac{\pi}{6}<\frac{3 \pi}{2}<4+\frac{\pi}{4}$. Следовательно,

$$
\sup _{(t, x) \in D}\left|\frac{\partial}{\partial x_{1}}\left(\sqrt{2} \cos \left(t+x_{1}\right)\right)\right|=\sup _{(t, x) \in D}\left|\sqrt{2} \sin \left(t+x_{1}\right)\right|=\left.\left|\sqrt{2} \sin \left(t+x_{1}\right)\right|\right|_{\substack{t=4, x_{1}=\frac{3 \pi}{2}-4}}=\sqrt{2} .
$$

На отрезке $\left[3+\frac{\pi}{6} ; 4+\frac{\pi}{4}\right]$ функция $\cos \left(t+x_{1}\right)$ монотонно возрастает. Поэтому максимум модуля этой функции достигается в одном из концов отрезка. Из того, что $4+\frac{\pi}{4}<\frac{7 \pi}{4}$ и $\frac{5 \pi}{4}<3+\frac{\pi}{6}$, имеем

$$
\left|\cos \left(4+\frac{\pi}{4}\right)\right|<\left|\cos \frac{7 \pi}{4}\right|=\frac{\sqrt{2}}{2}=\left|\cos \frac{5 \pi}{4}\right|<\left|\cos \left(3+\frac{\pi}{6}\right)\right|
$$

В результате

$$
\begin{gathered}
\sup _{(t, x) \in D}\left|\frac{\partial}{\partial x_{1}}\left(\sqrt{2} \sin \left(t+x_{1}\right)\right)\right|=\sup _{(t, x) \in D}\left|\sqrt{2} \cos \left(t+x_{1}\right)\right|=\left|\sqrt{2} \cos \left(t+x_{1}\right)\right|||_{\substack{t=3, x_{1}=\frac{\pi}{6}}} \\
=\sqrt{2}\left|\cos \left(3+\frac{\pi}{6}\right)\right|=\sqrt{2} \cos \left(3-\frac{5 \pi}{6}\right) .
\end{gathered}
$$

Таким образом,

$$
\left\|B\left(t, x^{(1)}\right) u-B\left(t, x^{(2)}\right) u\right\| \leq K \cdot d_{P}\left\|x^{(1)}-x^{(2)}\right\|=\sqrt{2+2 \cos ^{2}\left(3-\frac{5 \pi}{6}\right)} \cdot d_{P}\left\|x^{(1)}-x^{(2)}\right\| .
$$

В случае, когда $P=\left\{\left(u_{1}, u_{2}\right):\left|u_{1}\right| \leq 1,\left|u_{2}\right| \leq 1\right\}, d_{P}=2 \sqrt{2}$ и константа Липшица равна $\sqrt{16+16 \cos ^{2}\left(3-\frac{5 \pi}{6}\right)} ;$ а в случае, когда $P=\left\{\left(u_{1}, u_{2}\right): u_{1}^{2}+u_{2}^{2} \leq 1\right\}, d_{P}=2$ и константа Липшица равна $\sqrt{8+8 \cos ^{2}\left(3-\frac{5 \pi}{6}\right)}$.

И наконец, вычислим модуль непрерывности $\omega_{D}^{*}(\delta)$.

Зафиксируем некоторое $\delta \geq 0$. Пусть точки $\left(t^{\prime}, x^{\prime}\right),\left(t^{\prime \prime}, x^{\prime \prime}\right)$ таковы, что $\left|t^{\prime}-t^{\prime \prime}\right|+\left\|x^{\prime}-x^{\prime \prime}\right\| \leq \delta$. Пусть также $\left\|x^{\prime}-x^{\prime \prime}\right\|=m \delta$ при некотором $0 \leq m \leq 1$.

$$
\left\|B\left(t^{\prime}, x^{\prime}\right)-B\left(t^{\prime \prime}, x^{\prime \prime}\right)\right\|^{2}=2\left(\sin \left(t^{\prime}+x_{1}^{\prime}\right)-\sin \left(t^{\prime \prime}+x_{1}^{\prime \prime}\right)\right)^{2}+2\left(\cos \left(t^{\prime}+x_{1}^{\prime}\right)-\cos \left(t^{\prime \prime}+x_{1}^{\prime \prime}\right)\right)^{2}
$$




$$
+\left(\sin x_{1}^{\prime}-\sin x_{1}^{\prime \prime}\right)^{2}+\left(\cos x_{1}^{\prime}-\cos x_{1}^{\prime \prime}\right)^{2}=6-4 \cos \left(t^{\prime}-t^{\prime \prime}+x_{1}^{\prime}-x_{1}^{\prime \prime}\right)-2 \cos \left(x_{1}^{\prime}-x_{1}^{\prime \prime}\right) .
$$

Заметим, что $\max _{\substack{\left(t^{\prime}, x^{\prime}\right) \in D,\left(t^{\prime \prime}, x^{\prime \prime}\right) \in D}}\left\{\left|t^{\prime}-t^{\prime \prime}+x_{1}^{\prime}-x_{1}^{\prime \prime}\right|\right\}=1+\frac{\pi}{12}<\frac{\pi}{2}$. Из этого следует, что значения косинусов в формуле (3.17) положительны, и поэтому

$$
\left\|B\left(t^{\prime}, x^{\prime}\right)-B\left(t^{\prime \prime}, x^{\prime \prime}\right)\right\|^{2} \leq 6-4 \cos \delta-2 \cos (m \delta) .
$$

Пусть $\delta \geq 1+\frac{\pi}{12}$, тогда

$$
\left\|B\left(t^{\prime}, x^{\prime}\right)-B\left(t^{\prime \prime}, x^{\prime \prime}\right)\right\|^{2} \leq 6-4 \cos \left(1+\frac{\pi}{12}\right)-2 \cos \frac{\pi}{12} .
$$

Пусть $0 \leq \delta<1+\frac{\pi}{12}$. Найдем значение $m$, при котором величина $6-4 \cos \delta-2 \cos (m \delta)-$ максимальна. При указанном выборе параметра $\delta$ значение $\cos (m \delta)$ монотонно убывает с ростом $m$. Таким образом,

$$
\left\|B\left(t^{\prime}, x^{\prime}\right)-B\left(t^{\prime \prime}, x^{\prime \prime}\right)\right\|^{2} \leq 6-4 \cos \delta-2 \cos \delta=6-6 \cos \delta .
$$

В результате имеем

$$
\begin{aligned}
& \omega_{D}^{*}(\delta)=\max _{\substack{\left(t^{\prime}, x^{\prime}\right) \in D,\left(t^{\prime \prime}, x^{\prime \prime}\right) \in D}}\left\{\left\|F\left(t^{\prime}, x^{\prime}\right)-F\left(t^{\prime \prime}, x^{\prime \prime}\right)\right\|:\left|t^{\prime}-t^{\prime \prime}\right|+\left\|x^{\prime}-x^{\prime \prime}\right\| \leq \delta\right\} \\
& =\max _{\begin{array}{c}
\left(t^{\prime}, x^{\prime}\right) \in D, \\
\left(t^{\prime \prime}, x^{\prime \prime}\right) \in D
\end{array}}\left\{\left\|B\left(t^{\prime}, x^{\prime}\right) u-B\left(t^{\prime \prime}, x^{\prime \prime}\right) u\right\|:\left|t^{\prime}-t^{\prime \prime}\right|+\left\|x^{\prime}-x^{\prime \prime}\right\| \leq \delta\right\} \\
& =\max _{\substack{\left(t^{\prime}, x^{\prime}\right) \in D,\left(t^{\prime \prime}, x^{\prime \prime}\right) \in D}}\left\{\left\|B\left(t^{\prime}, x^{\prime}\right)-B\left(t^{\prime \prime}, x^{\prime \prime}\right)\right\|:\left|t^{\prime}-t^{\prime \prime}\right|+\left\|x^{\prime}-x^{\prime \prime}\right\| \leq \delta\right\} \cdot d_{P} \\
& =\left[\begin{array}{l}
\sqrt{6-4 \cos \left(1+\frac{\pi}{12}\right)-2 \cos \frac{\pi}{12}} \cdot d_{P}, \delta \geq 1+\frac{\pi}{12} \\
\sqrt{6-6 \cos \delta} \cdot d_{P}, 0 \leq \delta<1+\frac{\pi}{12},
\end{array}\right.
\end{aligned}
$$

где $d_{P}$ - диаметр множества $P$.

\section{Заключение}

В работе изучена задача о приближенном конструировании множеств достижимости нелинейных управляемых систем в конечномерных евклидовых пространствах. При не слишком ограничительных условиях на управляемую систему введены и рассмотрены нижние и верхние (по включению) аппроксимации множеств достижимости управляемой системы. Основу конструирования этих аппроксимаций составляет идеология ломаных Эйлера. С применением оценки Л. С. Понтрягина хаусдорфова отклонения выпуклого компакта в $\mathbb{R}^{m}$ от геометрической разности этого компакта и шара достаточно малого радиуса получены оценки отклонения нижних и верхних аппроксимаций множеств достижимости от (идеальных) множеств достижимости управляемой системы.

\section{СПИСОК ЛИТЕРАТУРЫ}

1. Куржанский А.Б. Управление и наблюдение в условиях неопределенности. М.: Наука, 1977. $392 \mathrm{c}$.

2. Куржанский А.Б. Избранные труды. М.: Изд-во МГУ, 2009. 756 с.

3. Kurzhanski A.B., Filippova T.F. On the theory of trajectory tubes: a mathematical formalism for uncertain dynamics, viability and control // Advances in Nonlinear Dynamics and Control / ed. A.B. Kurzhanski. Ser. PSCT 17. Boston: Birkhäuser, 1993. P. 122-188. 
4. Красовский Н. Н., Субботин А. И. Позиционные дифференциальные игры. М.: Наука, 1974. $456 \mathrm{c.}$

5. Понтрягин Л.С. Линейные дифференциальные игры преследования // Мат. сб. 1980. Т. 112 (154), № 3 (7). C. 307-330. doi: 10.1070/SM1981v040n03ABEH001815 .

6. Никольский М.С. Об аппроксимации множества достижимости для дифференциального включения // Вест. Москов. ун-та. Сер. 15. Вычисл. математика и кибернетика. 1987. №4. С. 31-34.

7. Черноусько Ф.Л. Оценивание фазового состояния динамических систем. М.: Наука, 1988. 319 с.

8. Kurzhanski A.B., Vályi I. Ellipsoidal techniques for dynamic systems: Control synthesis for uncertain systems // Dynamics and Control. 1992. Vol. 2, № 2. P. 87-111. doi: 10.1007/BF02169492 .

9. Овсеевич А.И., Черноусько Ф.Л. Двусторонние оценки областей достижимости управляемых систем // Прикл. математика и механика. 1982. Т. 46, вып. 5. С. 737-744. doi: 10.1016/0021-8928(82)90005-3.

10. Gusev M.I., Zykov I.V. On extremal properties of boundary points of reachable sets for a system with integrally constrained control // IFAC-PapersOnLine. 2017. Vol. 50, no. 1. P. 4082-4087. doi: $10.1016 /$ j.ifacol.2017.08.792 .

11. Veliov V. On the time-discretization of control systems // SIAM J. Control Optim. Vol. 35, no. 5. P. 1470-1486. doi: 10.1137/S0363012995288987.

12. Костоусова Е.К. Об ограниченности внешних полиэдральных оценок множеств достижимости линейных систем // Журн. вычисл. математики и мат. физики. 2008. Т. 48, №6. С. 974-989. doi: $10.1134 / \mathrm{S} 0965542508060043$.

13. Гусейнов Х.Г., Моисеев А.Н., Ушаков В.Н. Об аппроксимации областей достижимости управляемых систем // Прикл. математика и механика. 1998. Т. 62, № 2. С. 179-187. doi: 10.1016/S0021-8928(98)00022-7 .

14. Горнов А.Ю. Вычислительные технологии решения задач оптимального управления. Новосибирск: Наука, 2000. 297 с.

15. Лотов А.В. Новая внешняя оценка множества достижимости нелинейной многошаговой динамической системы // Журн. вычислит. математики и мат. физики. 2018. Т. 58, №2. С. 209-219. doi: $10.7868 / \mathrm{S} 0044466918020060$

16. Ушаков В.Н., Ухоботов В.И., Ушаков А.В., Паршиков Г.В. К решению задач о сближении управляемых систем // Тр. МИАН. 2015. Т. 291. С. 276-291. doi: 10.1134/S0371968515040214

Поступила 14.01.2020

После доработки 6.02.2020

Принята к публикации 10.02.2020

Ушаков Владимир Николаевич

д-р физ.-мат. наук, профессор

главный науч. сотрудник

Институт математики и механики им. Н. Н. Красовского УрО РАН

г. Екатеринбург

e-mail: ushak@imm.uran.ru

Першаков Максим Вадимович

математик

Институт математики и механики им. Н.Н. Красовского УрО РАН

г. Екатеринбург

e-mail: Mper192@yandex.ru

\section{REFERENCES}

1. Kurzhanski A.B. Upravlenie i nablyudenie v usloviyakh neopredelennosti [Control and observation under the conditions of uncertainty]. Moscow: Nauka Publ., 1977. 392 p.

2. Kurzhanski A.B. Izbrannye trudy (Selected works). Moscow: Moscow State University Publ., 2009, 756 p. (in Russian) 
3. Kurzhanski A.B., Filippova T.F. On the theory of trajectory tubes: a mathematical formalism for uncertain dynamics, viability and control. In: A.B. Kurzhanski (eds), Advances in Nonlinear Dynamics and Control. Ser. PSCT, vol. 17. Boston: Birkhäuser, 1993, pp. 122-188.

doi: 10.1007/978-1-4612-0349-0_4.

4. Krasovskii N.N., Subbotin A.I. Game-theoretical control problems. N Y: Springer, 1987, 517 p. This book is substantially revised version of the monograph Pozitsionnye differentsial'nye igry, Moscow, Nauka Publ., 1974, 456 p.

5. Pontryagin L.S. Linear differential games of pursuit. Math. USSR-Sb., 1981, vol. 40, no. 3, pp. $285-303$. doi: 10.1070/SM1981v040n03ABEH001815 .

6. Nikol'skii M.S. Approximation of the feasibility set for a differential inclusion. Vestnik Moskov. Univ. Ser. XV Vychisl. Mat. Kibernet., 1987, no. 4, pp. 31-34 (in Russian).

7. Chernous'ko F.L. Otsenivanie fasovogo sostoianiia dinamicheskih sistem [Estimation of the phase state of dynamical systems]. Moscow: Nauka Publ., 1988, 319 p. ISBN: 5-02-013899-1.

8. Kurzhanski A.B., Vályi I. Ellipsoidal techniques for dynamic systems: Control synthesis for uncertain systems. Dynamics and Control, 1992, vol. 2, no. 2, pp. 87-111. doi: 10.1007/BF02169492.

9. Ovseevich A.I., Chernous'ko F.L. Two-sided estimates on the attainability domains of cotrolled systems. J. Appl. Math. Mech., 1982, vol. 46, no. 5, pp. 590-595. doi: 10.1016/0021-8928(82)90005-3 .

10. Gusev M.I., Zykov I.V. On extremal properties of boundary points of reachable sets for a system with integrally constrained control. IFAC-PapersOnLine, 2017, vol. 50, no. 1, pp. 4082-4087. doi: 10.1016/j.ifacol.2017.08.792 .

11. Veliov V. On the time-discretization of control systems. SIAM J. Control Optim., 1997, vol. 35, no. 5, pp. 1470-1486. doi: 10.1137/S0363012995288987.

12. Kostousova E.K. On the boundedness of outer polyhedral estimates for reachable sets of linear systems. Comput. Math. and Math. Phys., 2008, vol. 48, no. 6, pp. 918-932. doi: 10.1134/S0965542508060043.

13. Guseinov K.G., Moiseyev A.A., Ushakov V.N. The approximation of reachable domains of control systems J. Appl. Math. Mech., 1998, vol. 62, no. 2, pp. 169-175. doi: 10.1016/S0021-8928(98)00022-7.

14. Gornov A.Yu. Vychislitel'nye tekhnologii resheniya zadach optimal'nogo upravleniya [The Computational Technologies for Solving Optimal Control Problems]. Novosibirsk: Nauka Publ., 2009, 278 p. ISBN: 978-5-02-023284-6.

15. Lotov A.V. New external estimate for the reachable set of a nonlinear multistep dynamic system. Comput. Math. Math. Phys., 2018, vol. 58, no. 2, pp. 196-206. doi: 10.7868/S0044466918020060.

16. Ushakov V.N., Ukhobotov V.I., Ushakov A.V., Parshikov G.V. On solving approach problems for control systems. Proc. Steklov Inst. Math., 2015, vol. 291, pp. 263-278. doi: 10.1134/S0371968515040214.

Received January 14, 2020

Revised February 6, 2020

Accepted February 10, 2020

Funding Agency: This work was supported by the Russian Foundation for Basic Research (projects no. 18-01-00264 and no. 18-01-00221).

Vladimir Nikolaevich Ushakov, Dr. Phys.-Math. Sci., RAS Corresponding Member, Prof., Krasovskii Institute of Mathematics and Mechanics of Ural Branch of the Russian Academy of Sciences, Yekaterinburg, 620108 Russia, e-mail: ushak@imm.uran.ru .

Maksim Vadimovich Pershakov, Krasovskii Institute of Mathematics and Mechanics of Ural Branch of the Russian Academy of Sciences, Yekaterinburg, 620108 Russia, e-mail: Mper192@yandex.ru .

Cite this article as: V.N. Ushakov, M.V.Pershakov. On two-sided approximations of reachable sets of control systems with geometric constraints on the controls, Trudy Instituta Matematiki $i$ Mekhaniki URO RAN, 2020, vol. 26, no. 1, pp. 239-255. 